\title{
Single name credit default swaptions meet single sided jump models
}

\author{
Henrik Jönsson • Wim Schoutens
}

(C) The Author(s) 2008. This article is published with open access at Springerlink.com

\begin{abstract}
Credit risk modeling is about modeling losses. These losses are typically coming unexpectedly and triggered by shocks. So any process modeling the stochastic nature of losses should reasonable include jumps. In this paper we review a few jump driven models for the valuation of Credit Default Swaps (CDSs) and show how under these dynamic models also pricing of (exotic) derivatives on single name CDSs is possible. More precisely, we set up fundamental firm-value models that allow for fast pricing of the 'vanillas' of the CDS derivative markets: payer and receiver swaptions. It turns out that the proposed model is able to produce realistic implied volatility smiles. Moreover, we detail how a CDS spread simulator can be set up under this framework and illustrate its use for the pricing of exotic derivatives with single name CDSs as underliers.
\end{abstract}

Keywords Single sided Levy processes · Structural models · Credit risk · Default probability $\cdot$ Credit Default Swaptions · Option pricing

JEL Classifications $\quad \mathrm{C} 02 \cdot \mathrm{G} 13$

\section{Introduction}

Credit Default Swaps (CDSs) have become in the last decennium very important instruments to deal with credit risk. These financial contracts are now available in

\author{
H. Jönsson $(\varangle)$ \\ EURANDOM, P.O. Box 513, 5600 MB Eindhoven, The Netherlands \\ e-mail: jonsson@eurandom.tue.nl \\ W. Schoutens \\ Department of Mathematics, K.U.Leuven, Leuven, Belgium
}


quite liquid form on thousands of underliers and are traded daily in huge volume. A market has been formed dealing with options or derivatives on these CDSs. The market is for the moment quite illiquid, but is expected to gain in volume over the next years.

Credit risk modeling is about modeling losses. These losses are typically coming unexpectedly and triggered by shocks. So any process modeling the stochastic nature of losses should reasonable include jumps. The presence of jumps is even of greater importance if one deals with derivatives on CDSs, because of the leveraging effects. Jump processes have already proven their modeling abilities in other settings like equity and fixed income (see Schoutens 2003) and have recently found their way into credit risk modeling. In this paper we review a few jump driven models for the valuation of CDSs and show how under these dynamic models also pricing of (exotic) derivatives on single name CDSs is possible. More precisely, we set up fundamental firm-value models that allow for fast pricing of the 'vanillas' of the CDS derivative markets: payer and receiver swaptions. Moreover, we detail how a CDS spread simulator can be set up under this framework and illustrate its use for the pricing of exotic derivatives on single name CDSs as underliers.

The standard model available to price swaptions is Black's model and implied volatilities are often extracted out of market data in a completely similar way as for the classical Black-Scholes equity model. In the scarce available market data a volatility smile is typically present. The model proposed here is able to produce such a volatility smile.

The paper is organized as follows. In Sect. 2, we present the one-sided Lévy firm value model and give examples of possible choices of Lévy processes to use, namely the Gamma, the Inverse Gaussian and the CMY process. In Sect. 3, we discuss the pricing of receiver and payer swaptions, by mapping the firm value to the spread value. We show how dynamic spreads can be generated by mapping the firm value paths to spread paths and how this can be used to price exotic options by Monte Carlo techniques in Sect. 4. Section 5 deals with the pricing of American options.

\section{Firm's value models}

The starting point of the model is the approach originally presented by Black and Cox (1976). According to this approach an event of default occurs when the asset value of the firm crosses a deterministic barrier. This barrier corresponds to the recovery value of the firm's debt.

Black and Cox assumed a geometrical Brownian motion for the firm's value processes. It is however well known that due to the continuous path nature of Brownian motion and the fast decaying tails of the underlying Normal distribution, the model cannot represent a realistic behavior of short term default probabilities. Indeed, the Brownian motion needs a substantial amount of time to reach a low barrier. In order to overcome these shortcomings, many extensions have already been proposed. The CreditGrades $^{\text {TM }}$ model (RiskMetrics Group, Inc. 2002), for example, tries to lift the short time default probabilities by making the barrier stochastic. 
Here we use the same methodology as Black and Cox but work under exponential Lévy models. By doing that, due to the jump nature, not only realistic default probabilities can be produced but also the other problem of predictability of the default time is overcome. Different Lévy models (both firm value and intensity) for credit derivatives pricing were explored by Cariboni (2007).

\subsection{One-sided Lévy processes}

We first introduce some notation. Let $Y=\left\{Y_{t}, t \geq 0\right\}$ be a pure jump Lévy process that has only negative jumps, i.e., $Y$ is spectrally negative, has no Brownian component, and no drift, and let $X=\left\{X_{t}, t \geq 0\right\}$ be given by

$$
X_{t}=\mu t+Y_{t}, \quad t \geq 0
$$

where $\mu$ is a positive real number.

The Laplace transform of $X_{t}$

$$
E\left[\exp \left(z X_{t}\right)\right]=\exp \left(t \psi_{X}(z)\right)
$$

where $\psi_{X}(z)$ is the Lévy exponent of $X_{1}$, which by the Lévy-Khintchin representation has the form

$$
\psi_{X}(z)=\mu z+\int_{-\infty}^{0}\left(e^{z x}-1+z(|x| \wedge 1)\right) v(d x) .
$$

The Lévy measure $v(d x)$ satisfies the integrability condition

$$
\int_{-\infty}^{0}(|x| \wedge 1) v(d x)<\infty
$$

For the processes we consider in this paper the Lévy measure has a density and we can write $v(d x)=m(x) d x$, where $m(x)$ is the density function. For the general theory of Lévy processes see, for example, Bertoin (1996) and Sato (2000).

\subsection{Lévy firm's value models}

We thus work under a firm's value setting, where the fundamental process that we model is the value of the reference entity of a CDS, and we opt to model the firm value by exponential Lévy driven jump models. These Lévy models have already proven their modeling abilities in different fields of finance, but especially in the credit modeling setting we are absolutely convinced of the necessity of jumps in underlying process. 
So assume that $X=\left\{X_{t}, t \geq 0\right\}$ is a pure jump Lévy process. The (risk neutral) value of the firm at time $t$ is then modeled by

$$
V_{t}=V_{0} \exp \left(X_{t}\right), \quad t \geq 0
$$

and we work under an admissible pricing measure $Q$ such that $E_{Q}\left[V_{t}\right]=V_{0} \exp (r t)$, where $r$ is the risk-free interest rate. We will refer to the process $V$ as the firm's value process. For a given recovery rate $R$, default occurs the first time

$$
V_{t}=V_{0} \exp \left(X_{t}\right) \leq R V_{0}
$$

or, equivalently, if

$$
X_{t} \leq \log R
$$

Let us denote by $P(t)$ the risk-neutral survival probability, or in other words the probability of no-default, between 0 and $t$ :

$$
\begin{aligned}
P(t) & =P_{Q}\left(X_{s}>\log R, \text { for all } 0 \leq s \leq t\right) \\
& =P_{Q}\left(\min _{0 \leq s \leq t} X_{S}>\log R\right) \\
& =E_{Q}\left[1\left(\min _{0 \leq s \leq t} X_{s}>\log R\right)\right] \\
& =E_{Q}\left[1\left(\min _{0 \leq s \leq t} V_{s}>R V_{0}\right)\right]
\end{aligned}
$$

where we used the indicator function 1(A), which is equal to 1 if the event $A$ is true and zero otherwise; the subindex $Q$ refers to the fact that we are working in a risk-neutral setting.

Consider a CDS with maturity $T$ and a continuous spread $c$. The upfront price of this CDS, i.e., the present value of the future cash flows for the buyer of protection, is then given by

$$
C D S=(1-R)\left(-\int_{0}^{T} \exp (-r s) \mathrm{d} P(s)\right)-c \int_{0}^{T} \exp (-r s) P(s) \mathrm{d} s
$$

where $R$ is the asset specific recovery rate and $r$ is the default-free discount rate. Note that in case of a default event the protection buyer is receiving $(1-R)$ for every insured currency unit. From this, we find the par spread $C$ that makes the CDS price equal to zero: 


$$
\begin{aligned}
C & =\frac{(1-R)\left(-\int_{0}^{T} \exp (-r s) \mathrm{d} P(s)\right)}{\int_{0}^{T} \exp (-r s) P(s) \mathrm{d} s} \\
& =\frac{(1-R)\left(1-\exp (-r T) P(T)-r \int_{0}^{T} \exp (-r s) P(s) \mathrm{d} s\right)}{\int_{0}^{T} \exp (-r s) P(s) \mathrm{d} s} .
\end{aligned}
$$

During the life-time of the CDS, the protection seller receives the fair par spread $C$ on the insured amount as a compensation of the default risk taken.

As we can see the pricing of a CDS depends fully on the expression of the default probability of the firm. In recent years, the calculation of these default probabilities (or equivalently in this setting, the hitting time probabilities) under several Lévy driven models have been worked out. We mention especially two approaches where the calculations can be done very fast, namely the Partial Integral-Differential Equation (PIDE) approach and the double Laplace inversion approach based on the WienerHopf Factorization. The first one can deal with general Lévy processes and is worked out for the very popular Variance Gamma (VG) case in, for example, Cariboni and Schoutens (2007). The second approach is only tractable for spectrally one-sided processes, only allowing for negative jumps and can be found in Rogers (2000) and Madan and Schoutens (2008). However, one could argue that in contrast to stock price behavior where clearly up and down jumps are present, a firm tries to follow a steady growth (up trend) but is exposed to shocks (negative jumps). It thus seems quite natural to model the underlying firm's value in a default model by a process with a positive drift and allow only for negative jumps. In contrast to the double sided situation, where the solution of the PIDE takes typically a couple of seconds on an ordinary computer, the double Laplace inversion can be performed within a fraction of a second.

\subsection{Examples}

We present here the three well known examples of Lévy models with positive upward trend and negative jumps given by Madan and Schoutens (2008): the Shifted Gamma, the Shifted Inverse Gaussian, and the Shifted CMY processes.

\subsubsection{The shifted Gamma-model}

The density function of the Gamma distribution Gamma $(a, b)$ with parameters $a>0$ and $b>0$ is given by

$$
f_{\text {Gamma }}(x ; a, b)=\frac{b^{a}}{\Gamma(a)} x^{a-1} \exp (-x b), \quad x>0 .
$$

The characteristic function is given by

$$
\phi_{\text {Gamma }}(u ; a, b)=(1-\mathrm{i} u / b)^{-a}, \quad u \in \mathbb{R} .
$$


Table 1 Mean, variance, skewness, and kurtosis of the Gamma distribution

\begin{tabular}{ll}
\hline & $\operatorname{Gamma}(a, b)$ \\
\hline Mean & $a / b$ \\
Variance & $a / b^{2}$ \\
Skewness & $2 / \sqrt{a}$ \\
Kurtosis & $3(1+2 / a)$ \\
\hline
\end{tabular}

Clearly, this characteristic function is infinitely divisible. The Gamma-process $G=$ $\left\{G_{t}, t \geq 0\right\}$ with parameters $a, b>0$ is defined as the stochastic process which starts at zero and has stationary, independent Gamma-distributed increments. More precisely, the time enters in the first parameter: $G_{t}$ follows a Gamma $(a t, b)$ distribution.

The Lévy density of the Gamma process is given by

$$
m(x)=a \exp (-b x) x^{-1}, \quad x>0 .
$$

The properties of the Gamma $(a, b)$ distribution given in Table 1 can easily be derived from the characteristic function.

Note also that we have the following scaling property: if $X$ is Gamma $(a, b)$ then for $c>0, c X$ is $\operatorname{Gamma}(a, b / c)$.

Let us start with a Gamma-process $G=\left\{G_{t}, t \geq 0\right\}$ with parameters $a>0$ and $b>0$. As driving Lévy process (in a risk-neutral setting), we then take

$$
X_{t}=\mu t-G_{t}, \quad t \geq 0,
$$

where in this case $\mu=r-\log (\phi(-i))=r+a \log \left(1+b^{-1}\right)$. Thus, there is a deterministic up trend with random downward shocks coming from the Gamma process.

The characteristic exponent is in this case available in closed form

$$
\psi(z)=\mu z-a \log \left(1+z b^{-1}\right) .
$$

Calibrating the Shifted Gamma model to the market CDS quotes of BAE Systems on January 5, 2005 gives the parameters $a=1.2028$ and $b=5.9720$. The fit of the Shifted Gamma model on the market CDSs is shown in Fig. 1.

An extensive calibration study was performed by Madan and Schoutens (2008) and the fitting error was typically around 1-2 basis points per quote.

\subsubsection{The shifted IG-model}

The Inverse Gaussian $\operatorname{IG}(a, b)$ law with parameters $a>0$ and $b>0$ has characteristic function

$$
\phi_{I G}(u ; a, b)=\exp \left(-a\left(\sqrt{-2 \mathrm{i} u+b^{2}}-b\right)\right), \quad u \in \mathbb{R} .
$$

The IG-distribution is infinitely divisible and we define the IG-process $I=\left\{I_{t}\right.$, $t \geq 0\}$ with parameters $a, b>0$ as the process which starts at zero, has independent 


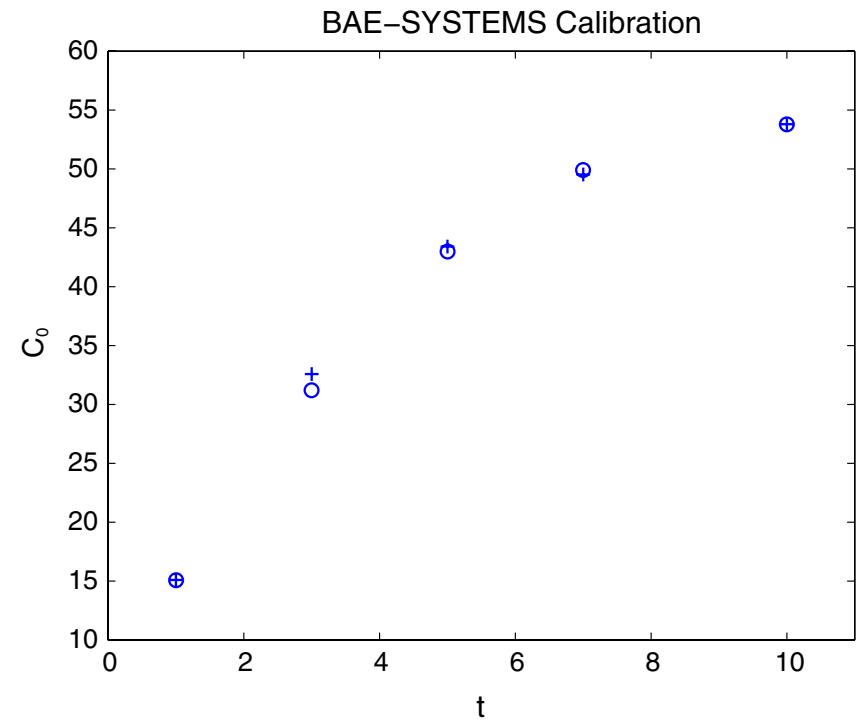

Fig. 1 Calibration BAE Systems. Underlying model is the Shifted Gamma with $a=1.2028$ and $b=$ 5.9720. Market spreads 'o' and model spreads '+'

Table 2 Mean, variance, skewness, and kurtosis of the Inverse Gaussian distribution

\begin{tabular}{ll}
\hline & $\operatorname{IG}(a, b)$ \\
\hline Mean & $a / b$ \\
Variance & $a / b^{3}$ \\
Skewness & $3 / \sqrt{a b}$ \\
Kurtosis & $3\left(1+5(a b)^{-1}\right)$ \\
\hline
\end{tabular}

and stationary IG-distributed increments, and such that

$$
\mathbb{E}\left[\exp \left(\mathrm{i} u I_{t}\right)\right]=\phi_{I G}(u ; a t, b)=\exp \left(-a t\left(\sqrt{-2 \mathrm{i} u+b^{2}}-b\right)\right), \quad u \in \mathbb{R}
$$

meaning that $I_{t}$ follows an $\operatorname{IG}(a t, b)$ distribution.

The density function of the $\operatorname{IG}(a, b)$ law is explicitly known

$$
f_{I G}(x ; a, b)=\frac{a}{\sqrt{2 \pi}} \exp (a b) x^{-3 / 2} \exp \left(-\left(a^{2} x^{-1}+b^{2} x\right) / 2\right), \quad x>0 .
$$

The Lévy density of the IG process is given by

$$
m(x)=\frac{a}{\sqrt{2 \pi}} \exp \left(-\frac{1}{2} b^{2} x\right) x^{-3 / 2}, \quad x>0 .
$$

The characteristics given in Table 2 can easily be obtained from the characteristic function. 
Let us start with an IG-process $I=\left\{I_{t}, t \geq 0\right\}$ with parameters $a>0$ and $b>0$. In our model, we then take

$$
X_{t}=\mu t-I_{t}, \quad t \geq 0,
$$

where in this case $\mu=r-\log (\phi(-i))=r+a\left(\sqrt{2+b^{2}}-b\right)$. We hence again have a deterministic up-trend and negative shocks that now are coming from an Inverse Gaussian process. We refer to this models as the Shifted IG model.

The characteristic exponent is in this case

$$
\psi(z)=\mu z-a\left(\sqrt{2 z+b^{2}}-b\right) .
$$

For calibration results on the Shifted IG model see Madan and Schoutens (2008).

\subsubsection{The shifted CMY-model}

The $\operatorname{CMY}(C, M, Y)$ distribution with parameters $C>0, M>0$, and $Y<1$ has characteristic function

$$
\phi_{C M Y}(u ; C, M, Y)=\exp \left\{C \Gamma(-Y)\left[(M-i u)^{Y}-M^{Y}\right]\right\}, \quad u \in \mathbb{R} .
$$

The Lévy density of the CMY process is given by

$$
m(x)=C \exp (-M x) x^{-1-Y}, \quad x>0
$$

It can be observed from the Gamma and IG Lévy densities that the corresponding Lévy processes are special cases of the CMY process: take $Y=0$ and $Y=1 / 2$, respectively.

Clearly, the CMY distribution is infinitely divisible. We define the CMY Lévy process $C=\left\{C_{t}, t \geq 0\right\}$ with parameters $C, M>0$ and $Y<1$ as the process that starts at zero and has stationary and independent CMY-distributed increments. More precisely $C_{t}$ follows a $\mathrm{CMY}(C t, M, Y)$ distribution. The properties of the $\operatorname{CMY}(C, M, Y)$ distribution given in Table 3 can be derived from its characteristic function.

Table 3 Mean, variance, skewness, and kurtosis of the CMY distribution

\begin{tabular}{ll}
\hline & $\operatorname{CMY}(C, M, Y)$ \\
\hline Mean & $C M^{Y-1} \Gamma(1-Y)$ \\
Variance & $C M^{Y-2} \Gamma(2-Y)$ \\
Skewness & $\frac{C M^{Y-3} \Gamma(3-Y)}{\left(C M^{Y-2} \Gamma(2-Y)\right)^{3 / 2}}$ \\
Kurtosis & $3+\frac{C M^{Y-4} \Gamma(4-Y)}{\left(C M^{Y-2} \Gamma(2-Y)\right)^{2}}$ \\
\hline
\end{tabular}


Let us start with a CMY-process $C=\left\{C_{t}, t \geq 0\right\}$ with parameters $C, M>0$ and $Y<1$. As driving Lévy process we take

$$
X_{t}=\mu t-C_{t}, \quad t \geq 0
$$

where in this case $\mu=r-\log (\phi(-i))=r-C \Gamma(-Y)\left((M+1)^{Y}-M^{Y}\right)$. Again there is a deterministic up trend with negative shocks now coming from a CMY process.

The characteristic exponent is in this case

$$
\psi(z)=\mu z+C \Gamma(-Y)\left((M+z)^{Y}-M^{Y}\right) .
$$

For calibration results on the Shifted CMY model see Madan and Schoutens (2008).

\section{Options on CDSs}

The firm's value approach is ideally suitable to set up a methodology for the pricing of options on CDSs. The most common options are payer and receiver swaptions. They are the counterpart of the classical European call and put options in other markets.

A receiver swaption holder on a single-name CDS has the right, but not the obligation, to sell protection at the strike spread level on expiry, in return for buying protection on the same CDS at market spread level on expiry. A payer swaption holder on a single-name CDS has the right, but not the obligation, to buy protection on the underlying CDS at the strike level on expiry, in return for selling protection at market spread at expiry. Special rules apply for the case when default happens before the swaption's maturity. Most common situation on the single-name payer and receiver structures is a knock-out clause, meaning that in case of early default the payoff is zero.

From the above it is clear that options on CDSs can be viewed as either options on CDS upfront prices or, equivalently, as options on CDS spreads.

\subsection{Swaption valuation}

Suppose we have a particular (Lévy driven) firm's value model under consideration and want to price options on CDSs under that model. As explained above the par spread under such a model depends on the following parameters:

- the initial firm value $V_{0}$;

- the recovery rate $R$, which also implies the default barrier $V_{0} R$;

- the risk-free rate $r$;

- the time of maturity of the CDS $T$;

- the parameters of the underlying Lévy process, which we denote by the vector $\theta$.

Hence, let us write for the fair par spread (at time zero):

$$
C_{0}=C\left(V_{0}, R, r, T, \theta\right) .
$$


Similarly we write:

$$
C D S_{0}\left(V_{0}, R, r, T, \theta, c\right)
$$

for the price to enter into a CDS agreement at time zero for a contract paying $c$ premium. Note that $C D S_{0}\left(V_{0}, R, r, T, \theta, C_{0}\right)=0$.

Consider an European option on a CDS and denote the maturity of this option by $T^{*}$. For the standard examples of the payer and receiver swaption, the payoff depend on the spread value at $T^{*}$ and in general the payoff can depend on the full evolution of the spread until the option's maturity, $T^{*}$. Hence, it is important to have a (stochastic) model for the spread at a future time point or more general on the evolution of the spread over a given time period.

Such a model can be readily set up under a firm's value approach by mapping it back to the evolution of the firm value. More precisely, we will propose the following stochastic model for the spread evolution over time:

$$
C_{t}=C\left(V_{t}, R, r, T, \theta\right), \quad t \geq 0 .
$$

Similarly, we also have the evolution of the upfront price

$$
C D S_{t}(c)=C D S_{t}\left(V_{t}, R, r, T, \theta, c\right), \quad t \geq 0 .
$$

Again, we have that

$$
C D S_{t}\left(C_{t}\right)=C D S_{t}\left(V_{t}, R, r, T, \theta, C_{t}\right)=0, \quad t \geq 0
$$

We will ignore in the modeling of the par spread the situation of early default and take this immediately into account in the options payoff.

Let us write $F\left(\left\{C D S_{t}, 0 \leq t \leq T^{*}\right\}, 1\left(\tau<T^{*}\right)\right)$ for the payoff function to indicate the that payoff can depend on the full path of the CDS' upfront price and on whether default occurred early $1\left(\tau<T^{*}\right)$ or not.

The price of an option is by risk-neutral valuation theory given by

$$
\Pi_{0}=\exp \left(-r T^{*}\right) E_{Q}\left[F\left(\left\{C D S_{t}, 0 \leq t \leq T^{*}\right\}, 1\left(\tau<T^{*}\right)\right)\right] .
$$

For a knock-out receiver and payer with strike spread $K$ this simplifies to respectively

$$
\begin{aligned}
& \Pi_{0}^{R}\left(T^{*}, K\right)=\exp \left(-r T^{*}\right) E_{Q}[\left(C D S_{T^{*}}\left(V_{T^{*}}, R, r, T, \theta, C_{T^{*}}\right)\right. \\
&\left.\left.\quad-C D S_{T^{*}}\left(V_{T^{*}}, R, r, T, \theta, K\right)\right)^{+} 1\left(\tau>T^{*}\right)\right] \\
&=\exp \left(-r T^{*}\right) E_{Q}\left[\left(-C D S_{T^{*}}\left(V_{T^{*}}, R, r, T, \theta, K\right)\right)^{+} \mid \tau>T^{*}\right] P\left(T^{*}\right)
\end{aligned}
$$


and

$$
\begin{aligned}
& \Pi_{0}^{P}\left(T^{*}, K\right)=\exp \left(-r T^{*}\right) E_{Q}[\left(C D S_{T^{*}}\left(V_{T^{*}}, R, r, T, \theta, K\right)\right. \\
&\left.\left.\quad-C D S_{T^{*}}\left(V_{T^{*}}, R, r, T, \theta, C_{T^{*}}\right)\right)^{+} 1\left(\tau>T^{*}\right)\right] \\
&=\exp \left(-r T^{*}\right) E_{Q}\left[\left(C D S_{T^{*}}\left(V_{T^{*}}, R, r, T, \theta, K\right)\right)^{+} \mid \tau>T^{*}\right] P\left(T^{*}\right) .
\end{aligned}
$$

Note that we can equivalently write the payoffs as functions of the par spread and the strike spread. The price of the knock-out receiver and payer is then given by, respectively,

$$
\Pi_{0}^{R}\left(T^{*}, K\right)=\exp \left(-r T^{*}\right) E_{Q}\left[A_{T^{*}}(T)\left(K-C_{T^{*}}\right)^{+} 1\left(\tau>T^{*}\right)\right]
$$

and

$$
\Pi_{0}^{P}\left(T^{*}, K\right)=\exp \left(-r T^{*}\right) E_{Q}\left[A_{T^{*}}(T)\left(C_{T^{*}}-K\right)^{+} 1\left(\tau>T^{*}\right)\right]
$$

where $A_{T^{*}}(T)$ is the risky annuity, i.e., the present value of a one basis point premium leg, from option maturity $T^{*}$ to CDS maturity $T$, evaluated at time $T^{*}$.

Cleverly studying and implementing the PIDE algorithm for the general Lévy case or the double Laplace inversion method for spectrally negative processes, learns that it is actually not really computationally more intensive to calculate $C\left(V_{0} \exp (x), R\right.$, $r, T, \theta)$ or, for that case, $C D S\left(V_{0} \exp (x), R, r, T, \theta, K\right)$, for a whole range of $x$ 's.

In Fig. 2 one finds the prices for 1 year receiver options on the BAE Systems CDS with 5 year maturity for a whole range of strike spreads calculated using the Shifted

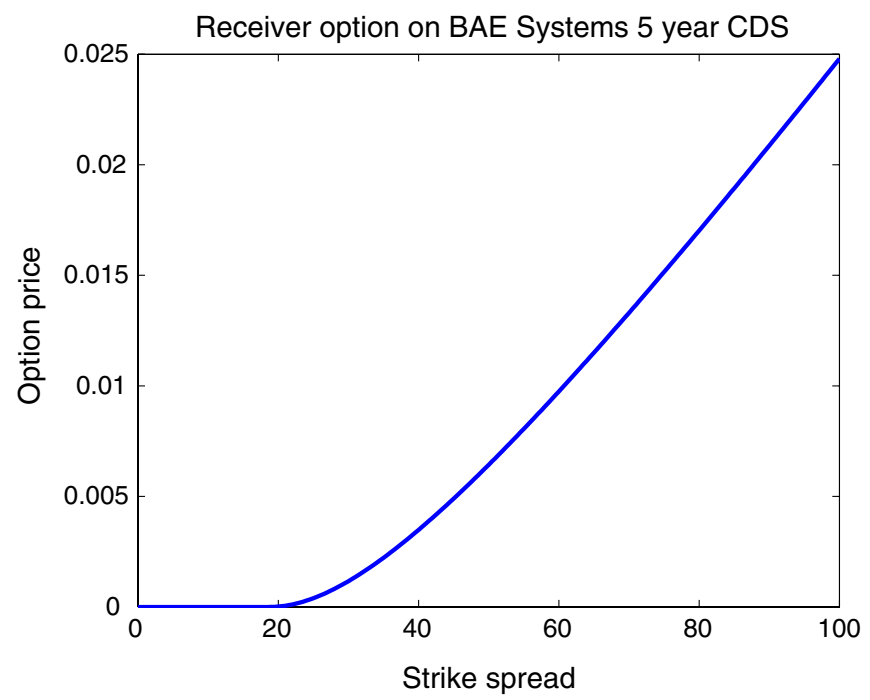

Fig. 2 One year receiver option prices on BAE Systems CDS with 5 year maturity. Underlying model is the Shifted Gamma with $a=1.2028$ and $b=5.9720$. The spot (par) spread is $C_{0}=43$ basis points and forward spread is $47 \mathrm{bp}$ 
Gamma model with parameters $a=1.2028$ and $b=5.9720$ given by calibrating the model to the BAE Systems spread term structure on January 5, 2005.

\subsection{Black's formulas and implied volatility}

The market standard for pricing options on CDSs are the Black formulas, based on the assumption that the credit spread follows a Geometric Brownian motion.

Before we write down the formulas let us first introduce some notation. The risky annuity, i.e., the present value of the premium leg assuming a one basis point premium, at valuation date $t=0$ to maturity $T$ is denoted by $A_{0}(T)$. That is

$$
A_{0}(T):=\int_{0}^{T} \exp (-r s) P(s) d s
$$

We denote by $A_{0}\left(T^{*}, T\right)$ the forward risky annuity at $t=0$ from option maturity $T^{*}$ to CDS maturity $T$. Note that $A_{0}\left(T^{*}, T\right)=A_{0}(T)-A_{0}\left(T^{*}\right)$.

A forward starting CDS is an agreement today to enter into a CDS contract at a time in the future. A long position in a forward starting CDS with start date $T_{1}$ and maturity $T_{2}$ is identical to enter into a long position in a CDS with maturity $T_{2}$ and a short position in a CDS with maturity $T_{1}$ on the same reference entity, i.e., buying protection for $T_{2}$ years and selling protection for $T_{1}$ years. This will exactly replicate the premium leg and the loss leg of the forward starting CDS for the time period $\left(0, T_{2}\right]$. The forward spread $F_{0}\left(T_{1}, T_{2}\right)$ is the fair spread agreed upon today for entering into a forward starting CDS starting at $T_{1}$ with maturity $T_{2}$ and is given by

$$
F_{0}\left(T_{1}, T_{2}\right)=\frac{C_{0}\left(T_{2}\right) A_{0}\left(T_{2}\right)-C_{0}\left(T_{1}\right) A_{0}\left(T_{1}\right)}{A_{0}\left(T_{2}\right)-A_{0}\left(T_{1}\right)},
$$

where $C_{0}(t):=C_{0}\left(V_{0}, R, r, t, \theta\right)$.

We can now, under the assumption that the CDS par spread at option maturity $T^{*}$ is the forward spread $F_{0}\left(T^{*}, T\right)$ times a log-normal random variable, write down the Black formulas for payer and receiver swaptions, respectively,

$$
\Pi_{0}^{P}\left(T^{*}, K\right)=A_{0}\left(T^{*}, T\right)\left(F_{0}\left(T^{*}, T\right) N\left(d_{1}\right)-K N\left(d_{2}\right)\right)
$$

and

$$
\Pi_{0}^{R}\left(T^{*}, K\right)=A_{0}\left(T^{*}, T\right)\left(K N\left(-d_{2}\right)-F_{0}\left(T^{*}, T\right) N\left(-d_{1}\right)\right)
$$

where

$$
d_{1}=\frac{\log \left(F_{0}\left(T^{*}, T\right) / K\right)+\sigma^{2} T^{*} / 2}{\sigma \sqrt{T^{*}}} \text { and } d_{2}=d_{1}-\sigma \sqrt{T^{*}} \text {. }
$$


Table 4 European payer and receiver values and implied volatilities

\begin{tabular}{lllll}
\hline Strike (bp) & Payer & Implied vol (\%) & Receiver & Implied vol (\%) \\
\hline 40.0 & 0.003710 & 42.4 & 0.001025 & 57.9 \\
42.0 & 0.003414 & 52.0 & 0.001636 & 65.3 \\
44.0 & 0.003164 & 59.1 & 0.002293 & 71.4 \\
46.0 & 0.002948 & 64.8 & 0.002984 & 76.6 \\
48.0 & 0.002758 & 69.7 & 0.003702 & 81.3 \\
50.0 & 0.002589 & 73.9 & 0.004440 & 85.4 \\
\hline
\end{tabular}

The estimated values of European payers and receivers, respectively, with maturity 0.25 year to enter into a single name CDS (BAE Systems) with a five year maturity and the corresponding Black's implied volatilities. The par spread is $43 \mathrm{bp}$ and the forward spread is $47 \mathrm{bp}$. The parameters of the underlying Shifted Gamma model are $a=1.2028$ and $b=5.9720$

Given the forward spread and the risky annuities we can calculate the implied volatilities for payers and receivers with different strikes. Implied volatilities for payers and receivers written on the same underlying CDS are given in Table 4. We have assumed a flat term structure of the interest rates of $3 \%$. The values of the options are generated using the Shifted Gamma model $(a=1.2028$ and $b=5.9720)$.

\section{A CDS spread generator and the pricing of exotic options on CDS}

Using the same methodology of mapping spreads and firm values, it is straightforward to generate dynamic spreads if a generator is available for the firm value process.

In order to implement the method, first calibrate the model on a given term-structure of market spreads in order to find the model parameters $\theta$ that matches best the current market situation. Next, we precalculate for a fine grid $\left\{x_{1}, \ldots, x_{n}\right\}$ all corresponding spread values $\left\{C\left(V_{0} \exp \left(x_{i}\right), R, r, T, \theta\right), i=1, \ldots, n\right\}$. Once this is done we start generating paths of the firm value process. For each firm value $V_{t}=V_{0} \exp \left(X_{t}\right)$, the corresponding spread is obtained by interpolating in $X_{t}$ the grid $\left\{x_{1}, \ldots, x_{n}\right\}$ and its corresponding spread values $\left\{C\left(V_{0} \exp \left(x_{i}\right), R, r, T, \theta\right), i=1, \ldots, n\right\}$.

In Fig. 3, we see a path of a firm value process under the Shifted Gamma model and its corresponding spread evolution. The parameters of the Gamma model were obtained by a calibration on the spread structure of BAE Systems on the January 5 , $2005(a=1.2028$ and $b=5.9720)$.

Once a fast spread generator is implemented we are set to price by Monte Carlo methods all kinds of exotic European structures on the evolution of the spread of a single name CDS.

We illustrate this by pricing a combinations of exotic derivatives. More precisely, we price

- a knock-out lookback receiver with price:

$$
\mathrm{LB}=\exp \left(-r T^{*}\right) E_{Q}\left[\left(-C D S_{T^{*}}\left(V_{T^{*}}, R, r, T, \theta, \max _{0 \leq u \leq T^{*}} C_{u}\right)^{+} \mid \tau>T_{*}\right] P\left(T_{*}\right)\right.
$$




\section{Firm's value}

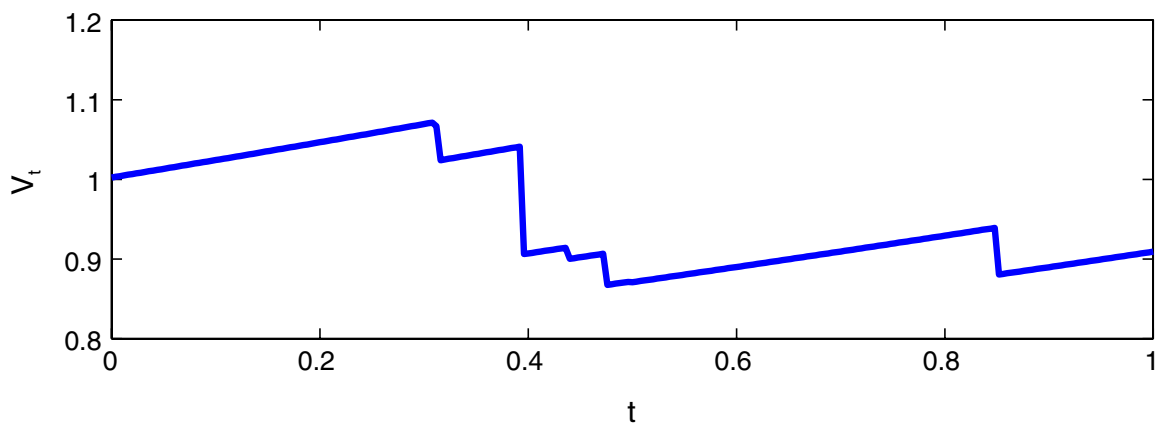

Spread

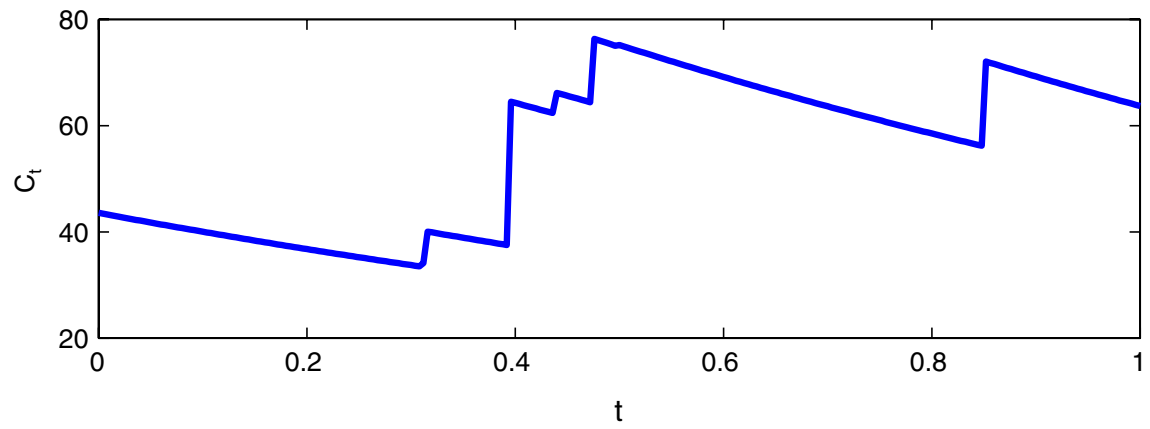

Fig. 3 Spread path—BAE Systems. Underlying model is the Shifted Gamma with $a=1.2028$ and $b=$ 5.9720

- $\quad$ a knock-out Variance Swap $\left(T^{*}=1, t_{i}=i / 252, i=0, \ldots, 252\right)$ :

$$
\mathrm{VS}=\exp \left(-r T^{*}\right) E_{Q}\left[\sum_{n=1}^{252}\left(\log \left(C_{t_{i}}\right)-\log \left(C_{t_{i-1}}\right)\right)^{2} \mid \tau>T_{*}\right] P\left(T_{*}\right)
$$

- a knock-out have-people-become-completely-crazy option with price:

$$
\begin{aligned}
& \text { CRAZY }=\exp \left(-r T^{*}\right) P\left(T_{*}\right) \\
& \times E_{Q}\left[\left(-C D S_{T^{*}}\left(V_{T^{*}}, R, r, T, \theta, \sin \left(\min _{\frac{T^{*}}{\pi}<u<T^{*}} \sqrt{C_{u}+0.01234}\right)\right)^{-\frac{3}{2}} / 10\right)^{+} \mid \tau>T_{*}\right] .
\end{aligned}
$$

The corresponding prices under 100, 000 Monte-Carlo simulations are given by

$$
\begin{aligned}
\text { LOOKBACK } & =0.01125 \\
\text { VS } & =0.00187 \\
\text { CRAZY } & =0.00425
\end{aligned}
$$

and were obtained on an ordinary PC in less than 5 min. 


\section{Regression based pricing of Bermudan swaptions}

With the approach to generate spread paths described above it is also possible to price American and Bermudan swaptions using the same methods as for options written on equities. For example, the regression based algorithms by Longstaff and Schwartz (2001) and Tsitsiklis and Van Roy $(1999,2001)$ can be adjusted to take into account the possibility that the option knock-out at the event of a default before option maturity and between exercise dates. An extensive overview of Monte Carlo methods in finance is given by Glasserman (2004).

Values for an European payer and Bermudan payers with semiannually, quarterly, and monthly exercise opportunities, respectively, all with a maturity of one year, to enter into a CDS with a fixed spread and five years maturity are shown in Table 5 for different strike spreads.

We used weighted Laguerre polynomials as a function of the spread value plus a constant as the basis functions for the regression. We could use other basis functions, such as powers of the state variable. We have, however, not noticed any difference in accuracy comparing the values and standard errors using different basis functions.

For a set of 100, 000 paths it takes approximately 25,30 , and 60 min to price the semiannual, the quarterly, and the monthly Bermudan option, respectively, for the range of strikes given in Table 5 .

To test the algorithm we have used the diagnostic test proposed in Longstaff and Schwartz (2001), namely, we first do the least squares estimation of the regression parameters based on one sample of paths and then use these estimates to price the option using a second sample of paths. The in-sample and out-of-sample option values do not differ significantly and the standard errors are of the same magnitude for both sets of prices.

Table 5 The estimated values of an European payer and Bermudan payers

\begin{tabular}{lllll}
\hline Strike $(\mathrm{bp})$ & European & Ber. semiann. & Ber. quarterly & Ber. monthly \\
\hline 44 & 0.009299 & 0.010305 & 0.012064 & 0.017081 \\
46 & 0.009007 & 0.009854 & 0.011290 & 0.015628 \\
48 & 0.008732 & 0.009450 & 0.010728 & 0.014597 \\
50 & 0.008473 & 0.009114 & 0.010256 & 0.013955 \\
52 & 0.008229 & 0.008802 & 0.009818 & 0.013075 \\
54 & 0.007999 & 0.008505 & 0.009428 & 0.012436 \\
56 & 0.007780 & 0.008223 & 0.009011 & 0.011781 \\
\hline
\end{tabular}

The estimated values of an European payer and Bermudan payers with semiannual, quarterly and monthly exercise, respectively, all with maturity one year to enter into a single name CDS with a five year maturity. The least squares estimate of the continuation value is based on the spread value. The simulation is based on 100,000 paths for the firm value process with 250 discretization points per year. The standard error of the estimates all lies between $0.86 * 10^{-4}$ and $1.25 * 10^{-4}$, with the smallest standard error for the European payer and the highest for the monthly Bermudan. Underlying model is the Shifted Gamma with $a=1.2028$ and $b=5.9720$ 


\section{Conclusions}

We showed how a CDS spread simulator based on a firm's value model driven by a single-sided Lévy process can be set up. The spread generator was used to price vanilla and exotic options on single name CDSs by employing Monte Carlo methods. Essentially, after calibrating on a CDS term structure, we simulate the firm value under the out of the calibration obtained jump dynamics. The paths of the firm value are then mapped into paths of the corresponding CDS spread. Out of this spread one can calculate straightforwardly the option payoff. We have priced in that way, the classical vanilla payers and receivers and have moreover calculated the corresponding (Black's) implied volatilities. It is important to note that the proposed models were capable to generate realistic implied volatility smiles. Next, we have illustrated the strength of the method by pricing several exotic payoff structures of European type. Further, we have used the already documented regression based techniques to price by Monte Carlo methods derivatives of American or Bermudan style under the current jump model setting. From a numerical point of view, the method is based on a double Laplace inversion together with the standard techniques of random number generation and, hence, the calculation of CDS spreads can be performed in terms of seconds on an ordinary computer. Further research goes into the direction of pricing Constant Maturity CDSs and other kinds of derivatives.

Acknowledgements H. Jönsson is an EU-Marie Curie Intra-European Fellow with funding from the European Community's Sixth Framework Programme (MEIF-CT-2006-041115).

Open Access This article is distributed under the terms of the Creative Commons Attribution Noncommercial License which permits any noncommercial use, distribution, and reproduction in any medium, provided the original author(s) and source are credited.

\section{References}

Bertoin, J. (1996). Lévy processes. Cambridge Tracts in Mathematics (Vol. 121). Cambridge: Cambridge University Press.

Black, F., \& Cox, J. C. (1976). Valuing corporate securities: Some effects on bond indenture provisions. Journal of Finance, 31, 351-367.

Cariboni, J. (2007). Credit derivatives pricing under Lévy models. PhD Thesis, Belgium: Katholieke Universiteit Leuven.

Cariboni, J., \& Schoutens, W. (2007). Pricing credit default swaps under Lévy models. Journal of Computational Finance, 10(4), 1-21.

Glasserman, P. (2004). Monte Carlo methods in financial engineering. Springer.

Longstaff, F. A., \& Schwartz, E. S. (2001). Valuing American options by simulation: A simple least squares approach. Review of Financial Studies, 14(1), 113-147.

Madan, D. B., \& Schoutens, W. (2008). Break on through to the single side. Journal of Credit Risk, 4(3), 3-20.

RiskMetrics Group, Inc. (2002). CreditGrades ${ }^{\mathrm{TM}}$, Technical Document.

Rogers, L. C. G. (2000). Evaluating first-passage probabilities for spectrally one-sided Lévy processes. Journal of Applied Probability, 37, 1173-1180.

Sato, K.-I. (2000). Lévy processes and infinitely divisible distributions. Cambridge Studies in Advanced Mathematics (Vol. 68). Cambridge: Cambridge University Press.

Schoutens, W. (2003). Lévy processes in finance: Pricing financial derivatives. Wiley. 
Tsitsiklis, J. N., \& Van Roy, B. (1999). Optimal stopping of Markov processes: Hilbert space theory, approximation algorithms, and an application to pricing high-dimensional financial derivatives. IEEE Transactions on Automatic Control, 44, 1840-1851.

Tsitsiklis, J. N., \& Van Roy, B. (2001). Regression methods for pricing complex American-style options. IEEE Transactions on Neural Networks, 12, 694-703. 